\title{
407.
}

\section{SECOND MEMOIR ON THE CURVES WHICH SATISFY GIVEN CONDITIONS; THE PRINCIPLE OF CORRESPONDENCE.}

[From the Philosophical Transactions of the Royal Society of London, vol. CLVIII. (for the year 1868), pp. 145-172. Received April 18,-Read May 2, 1867.]

Is the present Memoir I reproduce with additional developments the theory established in my paper "On the Correspondence of two points on a Curve" (London Math. Society, No. VII., April 1866), [385] ; and I endeavour to apply it to the determination of the number of the conics which satisfy given conditions; viz. these are conditions of contact with a given curve, or they may include arbitrary conditions $Z, 2 Z$, \&c. If, for a moment, we consider the more general question where the Principle is to be applied to finding the number of the curves $C^{r}$ of the order $r$, which satisfy given conditions of contact with a given curve, there are here two kinds of special solutions; viz., we may have proper curves $C^{r}$ touching (specially) the given curve at a cusp or cusps thereof, and we may have improper curves, that is, curves which break up into two or more curves of inferior orders. In the case where the curves $C^{r}$ are lines, there is only the first kind of special solution, where the sought for lines touch at a cusp or cusps. But in the case to which the Memoir chiefly relates, where the curves $C^{r}$ are conics, we have the two kinds of special solutions, viz., proper conics touching at a cusp or cusps, and conics which are line-pairs or point-pairs. In the application of the Principle to determining the number of the conics which satisfy any given conditions, I introduce into the equation a term called the "Supplement" (denoted by the abbreviation "Supp."), to include the special solutions of both kinds. The expression of the Supplement should in every case be furnished by the theory; and this being known, we should then have an equation leading to the number of the conics which properly satisfy the prescribed conditions; but in thus finding the expression of the Supplements, there are difficulties which I am unable to overcome; and I have contented myself with the reverse course, viz., knowing in each case the 
number of the proper solutions, I use these results to determine à posteriori in each case the expression of the Supplement; the expression so obtained can in some cases be accounted for readily enough, and the knowledge of the whole series of them will be a convenient basis for ulterior investigations.

The Principle of Correspondence for points in a line was established by Chasles in the paper in the Comptes Rendus, June-July 1864, referred to in my First Memoir; it is extended to unicursal curves in a paper of the same series, March 1866, "Sur les courbes planes ou à double courbure dont les points peuvent se déterminer individuellement-Application du Principe de Correspondance dans la théorie de ces courbes," but not to the case of a curve of given deficiency $D$ considered in my paper of April 1866 above referred to. The fundamental theorem in regard to unicursal curves, viz. that in a curve of the order $m$ with $\frac{1}{2}(m-1)(m-2)$ double points (nodes or cusps) the coordinates $(x, y, z)$ are proportional to rational and integral functions of a variable parameter $\theta$, - as a case of a much more general theorem of Riemann's-dates from the year 1857, but was first explicitly stated by Clebsch in the paper "Ueber diejenigen ebenen Curven deren Coordinaten rationale Functionen eines Parameters sind," Crelle, t. Lxiv. (1864), pp. 43-63. See also my paper "On the Transformation of Plane Curves," London Mathematical Society, No. III., Oct. 1865, [384].

The paragraphs of the present Memoir are numbered consecutively with those of the First Memoir.

\section{Article Nos. 94 to 104.-On the Correspondence of two points on a Curve.}

94. In a unicursal curve the coordinates $(x, y, z)$ of any point thereof are proportional to rational and integral functions of a variable parameter $\theta$. Hence if two points of the curve correspond in such wise that to a given position of the first point there correspond $\alpha^{\prime}$ positions of the second point, and to a given position of the second point $\alpha$ positions of the first point, the number of points which correspond each to itself is $=\alpha+\alpha^{\prime}$. For let the two points be determined by their parameters $\theta$, $\theta^{\prime}$ respectively, then to a given value of $\theta$ there correspond $\alpha^{\prime}$ values of $\theta^{\prime}$, and to a given value of $\theta^{\prime}$ there correspond $\alpha$ values of $\theta$; hence the relation between $\left(\theta, \theta^{\prime}\right)$ is of the form $(\theta, 1)^{a}\left(\theta^{\prime}, 1\right)^{a^{\prime}}=0$; and writing therein $\theta^{\prime}=\theta$, then for the points which correspond each to itself, we hav an equation $(\theta, 1)^{a+\alpha^{\prime}}=0$, of the order $\alpha+\alpha^{\prime}$; that is, the number of these points is $=\alpha+\alpha^{\prime}$.

Hence for a unicursal curve we have a theorem similar to that of M. Chasles' for a line, viz. the theorem may be thus stated:

If two points of a unicursal curve have an $\left(\alpha, \alpha^{\prime}\right)$ correspondence, the number of united points is $=\alpha+\alpha^{\prime}$. But a unicursal curve is nothing else than a curve with a deficiency $D=0$, and we thence infer:

THEOREM. If two points of a curve with deficiency $D$ have an $\left(\alpha, \alpha^{\prime}\right)$ correspondence, the number of united points is $=\alpha+\alpha^{\prime}+2 k D$; in which theorem $2 k$ is a coefficient to be determined. 
95. Suppose that the corresponding points are $P, P^{\prime}$ and imagine that when $P$ is given the corresponding points $P^{\prime}$ are the intersections of the given curve by a curve $\Theta$ (the equation of the curve $\Theta$ will of course contain the coordinates of $P$ as parameters, for otherwise the position of $P^{\prime}$ would not depend upon that of $P$ ). I find that if the curve $\Theta$ has with the given curve $k$ intersections at the point $P$, then in the system of points $\left(P, P^{\prime}\right)$ the number of united points is

$$
a=\alpha+\alpha^{\prime}+2 k D
$$

whence in particular if the curve $\Theta$ does not pass through the point $P$, then the number of united points is $=\alpha+\alpha^{\prime}$, as in the case of a unicursal curve. (I have in the paper of April 1866 above referred to, proved this theorem in the particular case where the $k$ intersections at the point $P$ take place in consequence of the curve $\Theta$ having a $k$-tuple point at $P$, but have not gone into the more difficult investigation for the case where the $k$ intersections arise wholly or in part from a contact of the curve $\Theta$, or any branch or branches thereof, with the given curve at $P$.)

96. It is to be observed that the general notion of a united point is as follows: taking the point $P$ at random on the given curve, the curve $\Theta$ has at this point $k$ intersections with the given curve; the remaining intersections are the corresponding points $P^{\prime}$; if for a given position of $P$ one or more of the points $P^{\prime}$ come to coincide with $P$, that is, if for the given position of $P$ the curve $\Theta$ has at this point more than $k$ intersections with the given curve, then the point in question is a united point.

It might at first sight appear that if for a given position of $P$ a number $2,3, \ldots$ or $j$ of the points $P^{\prime}$ should come to coincide with $P$, then that the point in question should reckon, for $2,3, \ldots$ or $j$ (as the case may be) united points: but this is not so. This is perhaps most easily seen in the case of a unicursal curve; taking the equation of correspondence to be $(\theta, 1)^{\alpha}\left(\theta^{\prime}, 1\right)^{\alpha^{\prime}}=0$, then we have $\alpha+\alpha^{\prime}$ united points corresponding to the values of $\theta$ which satisfy the equation $(\theta, 1)^{\alpha}(\theta, 1)^{\alpha^{\prime}}=0$; if this equation has a $j$-tuple root $\theta=\lambda$, the point $P$ which answers to this value $\lambda$ of the parameter is reckoned as $j$ united points. But starting from the equation $(\theta, 1)^{a}\left(\theta^{\prime}, 1\right)^{\alpha^{\prime}}=0$, if on writing in this equation $\theta=\lambda$, the resulting equation $(\lambda, 1)^{\alpha}\left(\theta^{\prime}, 1\right)^{\alpha^{\prime}}=0$ has a root $\theta^{\prime}=\lambda$, it follows that the equation $(\theta, 1)^{a}(\theta, 1)^{a^{\prime}}=0$ has a root $\theta=\lambda$, and that the point which belongs to the value $\theta=\lambda$ is a united point; if on writing in the equation $\theta=\lambda$, the resulting equation $(\lambda, 1)^{a}\left(\theta^{\prime}, 1\right)^{a^{\prime}}=0$ has a $j$-tuple root $\theta^{\prime}=\lambda$, it does not follow that the equation $(\theta, 1)^{\alpha}(\theta, 1)^{\alpha^{\prime}}=0$ has a $j$-tuple root $\theta=\lambda$, nor consequently that the point answering to $\theta=\lambda$ in anywise reckons as $j$ united points.

97. This may be further illustrated by regarding the parameters $\theta, \theta^{\prime}$ as the coordinates of a point in a plane; the equation $(\theta, 1)^{a}\left(\theta^{\prime}, 1\right)^{\alpha^{\prime}}=0$ is that of a curve of the order $\alpha+\alpha^{\prime}$, having an $\alpha$-tuple point at infinity on the axis $\theta=0$, and an $\alpha^{\prime}$-tuple point at infinity on the axis $\theta^{\prime}=0$; the united points are given as the intersections of the curve with the line $\theta=\theta^{\prime}$; a $j$-fold intersection, whether arising from a multiple point of the curve or from a contact of the line $\theta=\theta^{\prime}$ with the curve, c. VI. 
gives a point which reckons as $j$ united points. But if $\theta=\lambda$ gives the $j$-fold root $\theta^{\prime}=\lambda$, this shows that the line $\theta=\lambda$ has with the curve $j$ intersections at the point $\theta=\theta^{\prime}=\lambda$; not that the line $\theta=\theta^{\prime}$ has with the curve $j$ intersections at the point in question.

98. Reverting to the notion of a united point as a point $P$ which is such that one or more of the corresponding points $P^{\prime}$ come to coincide with $P$; in the case where $P$ is at a node of the given curve, it is necessary to explain that the point $P$ must be considered as belonging to one or the other of the two branches through the node, and that the point $P$ is not to be considered as a united point unless we have on the same branch of the curve one or more of the corresponding points $P^{\prime}$ coming to coincide with the point $P$. If, to fix the ideas, $k=1$, that is, if the curve $\Theta$ simply pass through the point $P$, then if $P$ be at a node the curve $\Theta$ passes through the node and has therefore at this point two intersections with the given curve; but the second intersection belongs to the other branch, and the node is not a united point; in order to make it so, it is necessary that the curve $\Theta$ should at the node touch the branch to which the point $P$ is considered to belong. The thing appears very clearly in the case of a unicursal curve; we have here two values $\theta=\lambda, \theta=\lambda^{\prime}$ answering to the node according as it is considered as belonging to one or the other branch of the curve; and in the equation of correspondence $(\theta, 1)^{a}\left(\theta^{\prime}, 1\right)^{a^{\prime}}=0$, writing $\theta=\lambda$, we have an equation $(\lambda, 1)^{\alpha}\left(\theta^{\prime}, 1\right)^{a^{\prime}}=0$ satisfied by $\theta^{\prime}=\lambda^{\prime}$ but not by $\theta^{\prime}=\lambda$, and the equation $(\theta, 1)^{a}(\theta, 1)^{a^{\prime}}=0$ is thus not satisfied by the value $\theta=\lambda$. The conclusion is that a node quì node is not a united point.

99. But it is otherwise as regards a cusp. When the point $P$ is at a cusp, the curve $\Theta$ (which has in general with the given curve $k$ intersections at $P$ ) has here more than $k$ intersections, and (as in this case there is no distinction of branch) the cusp reckons as a united point. In the case of a unicursal curve, there is at the cusp a single value $\theta=\lambda$ of the parameter, and the equation $(\theta, 1)^{\alpha}(\theta, 1)^{\alpha^{\prime}}=0$ is satisfied by the value $\theta=\lambda$. But for the very reason that the cusp quì cusp reckons as a united point, the cusp is a united point only in an improper or special sense, and it is to be rejected from the number of true united points. We may include the cusps, along with any other special solutions which may present themselves, under a head "Supplement," and instead of writing as above $a-\alpha-\alpha^{\prime}=2 k D$, write $a-\alpha-\alpha^{\prime}+$ Supp. $=2 k D$.

Before going further I apply the theorem to some examples in which the curve $\Theta$ is a system of lines.

100. Investigation of the class of a curve of the order $m$ with $\delta$ nodes and $\kappa$ cusps. Take as corresponding points on the given curve two points such that the line joining them passes through a fixed point $O$; the united points will be the points of contact of the tangents through $O$; that is, the number of the united points will be equal to the class of the curve. The curve $\Theta$ is here the line $O P$ which has with the given curve a single intersection at $P$; that is, we have $k=1$. The points $P^{\prime}$ corresponding to a given position of $P$ are the remaining $m-1$ intersections of $O P$ with the curve, that is, we have $\alpha^{\prime}=m-1$; and in like manner $\alpha=m-1$. Each of the 
cusps is (specially) a united point, and counts once, whence the Supplement is $=\kappa$. Hence, writing $n$ for the class, we have $n+2(m-1)+\kappa=2 D$, or writing for $2 D$ its value $=m^{2}-3 m+2-2 \delta-2 \kappa$, we have $n=m^{2}-m-2 \delta-3 \kappa$, which is right.

101. Investigation of the number of inflexions. Taking the point $P^{\prime}$ to be a tangential of $P$ (that is, an intersection of the curve by the tangent at $P$ ), the united puints are the inflexions; and the number of the united points is equal to the number of the inflexions. The curve $\Theta$ is the tangent at $P$ having with the given curve two intersections at this point; that is, $k=2 ; P^{\prime}$ is any one of the $m-2$ tangentials of $P$, that is, $\alpha^{\prime}=m-2$; and $P$ is the point of contact of any one of the $n-2$ tangents from $P^{\prime}$ to the curve, that is, $\alpha=n-2$. Each cusp is (specially) a united point, and counts once, whence the Supplement is $=\kappa$. Hence, writing $\iota$ for the number of inflexions, we have

$$
\iota-(m-2)-(n-2)+\kappa=4 D
$$

or substituting for $2 D$ its value expressed in the form $n-2 m+2+\kappa$, we have

which is right.

$$
\iota=3 n-3 m+\kappa,
$$

102. For the purpose of the next example it is necessary to present the fundamental equation under a more general form. The curve $\Theta$ may intersect the given curve in a system of points $P^{\prime}$, each $p$ times, a system of points $Q^{\prime}$, each $q$ times, \&c. in such manner that the points $\left(P, P^{\prime}\right)$, the points $\left(P, Q^{\prime}\right)$, \&c. are pairs of points corresponding to each other according to distinct laws; and we shall then have the numbers $\left(\mathrm{a}, \alpha, \alpha^{\prime}\right),\left(\mathrm{b}, \beta, \beta^{\prime}\right), \& \mathrm{c}$., corresponding to these pairs respectively, viz. $\left(P, P^{\prime}\right)$ are points having an $\left(\alpha, \alpha^{\prime}\right)$ correspondence, and the number of united points is $=a$; $\left(P, Q^{\prime}\right)$ are points having a $\left(\beta, \beta^{\prime}\right)$ correspondence, and the number of united points is $=b$, and so on. The theorem then is

$$
p\left(\mathrm{a}-\alpha-\alpha^{\prime}\right)+q\left(\mathrm{~b}-\beta-\beta^{\prime}\right)+\& \mathrm{c} .+ \text { Supp. }=2 k D,
$$

being in fact the most general form of the theorem for the correspondence of two points on a curve, and that which will be used in all the investigations which follow.

103. Investigation of the number of double tangents. Take $P^{\prime}$ an intersection of the curve with a tangent from $P$ to the curve (or, what is the same thing, $P, P^{\prime}$ cotangentials of any point of the curve): the united points are here the points of contact of the several double tangents of the curve; or if $\tau$ be the number of double tangents, then the number of united points is $=2 \tau$. The curve $\Theta$ is the system of the $n-2$ tangents from $P$ to the curve; each tangent has with the curve a single intersection at $P$, that is, $k=n-2$; each tangent besides meets the curve in the point of contact $Q^{\prime}$ twice, and in $(m-3)$ points $P^{\prime}$; hence if $\left(a, \alpha, \alpha^{\prime}\right)$ refer to the points $\left(P, Q^{\prime}\right)$, and $\left(2 \tau, \beta, \beta^{\prime}\right)$ to the points $\left(P, P^{\prime}\right)$, we have

$$
2\left\{a-\alpha-\alpha^{\prime}\right\}+\left\{2 \tau-\beta-\beta^{\prime}\right\}+\text { Supp. }=2(n-2) D .
$$

From the foregoing example the value of $a-\alpha-\alpha^{\prime}$ is $=4 D-\kappa$. In the case where $34-2$ 
the point $P$ is at a cusp, then the $n-2$ tangents become the $n-3$ tangents from the cusp, and the tangent at the cusp; hence the curve $\Theta$ meets the given curve in $2(n-3)+3,=2 n-3$ points, that is, $(n-2)+(n-1)$ points; this does not prove (ante, No. 96), but the fact is, that the cusp counts in the Supplement $(n-1)$ times, and the expression of the Supplement is $=(n-1) \kappa$. It is clear that we have $\beta=\beta^{\prime}=(n-2)(m-3)$, so that the equation is

that is

$$
8 D-2 \kappa+2 \tau-2(n-2)(m-3)+(n-1) \kappa=(n-2) 2 D,
$$

$$
2 \tau=2(n-2)(m-3)+(n-6) 2 D+(-n+3) \kappa ;
$$

or substituting for $2 D$ its value $=n-2 m+2+\kappa$ and reducing, this is

$$
2 \tau=n^{2}+8 m-10 n-3 \kappa,
$$

which is right.

104. As another example, suppose that the point $P$ on a given curve of the order $m$ and the point $Q$ on a given curve of the order $m^{\prime}$ have an $\left(\alpha, \alpha^{\prime}\right)$ correspondence, and let it be required to find the class of the curve enveloped by the line $P Q$. Take an arbitrary point $O$, join $O Q$, and let this meet the curve $m$ in $P^{\prime}$; then $\left(P, P^{\prime}\right)$ are points on the curve $m$ having a $\left(m^{\prime} \alpha, m \alpha^{\prime}\right)$ correspondence; in fact to a given position of $P$ there correspond $\alpha^{\prime}$ positions of $Q$, and to each of these $m$ positions of $P^{\prime}$; that is, to each position of $P$ there correspond $m \alpha^{\prime}$ positions of $P^{\prime}$; and similarly to each position of $P^{\prime}$ there correspond $m^{\prime} \alpha$ positions of $P$. The curve $\Theta$ is the system of the lines drawn from each of the $\alpha^{\prime}$ positions of $Q$ to the point $O$, hence the curve $\Theta$ does not pass through $P$, and we have $k=0$. Therefore the number of the united points $\left(P, P^{\prime}\right)$, that is, the number of the lines $P Q$ which pass through the point $O$, is $=m \alpha^{\prime}+m^{\prime} \alpha$, or this is the class of the curve enveloped by $P Q$.

It is to be noticed that if the two curves are curves in space (plane, or of double curvature), then the like reasoning shows that the number of the lines $P Q$ which meet a given line $O$ is $=m \alpha^{\prime}+m^{\prime} \alpha$, that is, the order of the scroll generated by the line $P Q$ is $=m \alpha^{\prime}+m^{\prime} \alpha$.

Article Nos. 105 to 111.-Application to the Conics which satisfy given conditions, one at least arbitrary.

105. Passing next to the equations which relate to a conic, we seek for $(4 Z)(1)$, the number of the conics which satisfy any four conditions $4 Z$ and besides touch a given curve, $(3 Z)(2)$ and $(3 Z)(1,1)$, the number of the conics which satisfy three conditions, and besides have with the given curve a contact of the second order, or (as the case may be) two contacts of the first order; and so on with the conditions $2 Z, Z$, and then finally $(5),(4,1), \ldots(1,1,1,1,1)$, the numbers of the conics which have with the given curve a contact of the fifth order, or a contact of the fourth and also of the first order..., or five contacts of the first order. 
106. As regards the case $(4 Z)(1)$, taking $P$ an arbitrary point of the given curve $m$, and for the curve $\Theta$ the system of the conics $(4 Z)(\overline{1})$ which pass through the given point $P$ and besides satisfy the four conditions, then the curve $\Theta$ has with the given curve $(4 Z)(\overline{1})$ intersections at $P$, and the points $P^{\prime}$ are the remaining $(2 m-1)(4 Z)(\overline{1})$ intersections: in the case of a united point $\left(P, P^{\prime}\right)$, some one of the system of conics becomes a conic $(4 Z)(1)$; and the number of the united points is consequently equal to that of the conics $(4 Z)(1)$; we have thus the equation

$$
\{(4 Z)(1)-2(2 m-1)(4 Z)(\overline{1})\}+\operatorname{Supp} .(4 Z)(\overline{1})=(4 Z)(\overline{1}) \cdot 2 D \text {. }
$$

107. It is in the present case easy to find $\grave{a}$ priori the expression for the Supplement. $1^{\circ}$. The system of conics $(4 Z)$ contains $2(4 Z \cdot)-(4 Z /)$ point-pairs $\left(^{1}\right)$; each of these, regarded as a line, meets the given curve in $m$ points, and each of these points is (specially) a united point $\left(P, P^{\prime}\right)$; this gives in the Supplement the term $m\{2(4 Z \cdot)-(4 Z /)\} .2^{\circ}$. The number of the conics $(4 Z)$ which can be drawn through a cusp of the given curve is $=(4 \dot{Z} \cdot)$; and the cusp is in respect of each of these conics a united point; we have thus the term $\kappa(4 Z \cdot)$, and the Supplement is thus $=m\{2(4 Z \cdot)-(4 Z /)\}+\kappa(4 Z \cdot)$. We have moreover $(4 Z)(\overline{1})=(4 Z \cdot), 2 D=n-2 m+2+\kappa$; and substituting these values, we find

$$
\begin{aligned}
(4 Z)(1)= & (4 m-2)(4 Z \cdot) \\
& -m\{2(4 Z \cdot)-(4 Z /)\}-\kappa(4 Z \cdot) \\
& +(n-2 m+2+\kappa)(4 Z \cdot) \\
= & n(4 Z \cdot)+m(4 Z /),
\end{aligned}
$$

which is right.

108. It is clear that if, instead of finding as above the expression of the Supplement, the value of $(4 Z)(1),=n(4 Z \cdot)+m(4 Z /)$, had been taken as known, then the equation would have led to

$$
\text { Supp. }(4 Z)(\overline{1})=m\{2(4 Z \cdot)-(4 Z /)\}+\kappa(4 Z \cdot) ;
$$

and this, as in fact already remarked, is the course of treatment employed in the remaining cases. It is to be observed also that the equation may for shortness be written in the form

$$
\text { (4Z) } \begin{aligned}
\{(1)- & 2(2 m-1)(\overline{1})\} \\
& + \text { Supp. }(\overline{1})=(\overline{1}) 2 D ;
\end{aligned}
$$

viz. the $(4 Z)$ is to be understood as accompanying and forming part of each symbol; and the like in other cases.

109. We have the series of equations

(4Z) $\{(1)-(\overline{1})(2 m-1)-(\overline{1})(2 m-1)\}$

+ Supp. $(\overline{1})$

$$
=(\overline{1}) 2 D ;
$$

1 The expression a point-pair is regarded as equivalent to and standing for that of a coincident line-pair: see First Memoir, No. 30. 
(3Z) $\quad\{(2)-(\overline{2})(2 m-2)-(\overline{1}, 1)\}$

+ Supp. $(\overline{2})$

$=2(\overline{2}) 2 D ;$

(3Z) $2\{(2)-(\overline{1}, 1)-(\overline{2})(2 m-2)\}$

$+\{2(1,1)-(\overline{1}, 1)(2 m-3)-(\overline{1}, 1)(2 m-3)\}$

+ Supp. $(\overline{1}, 1)$

$=(\overline{1}, 1) 2 D$;

(2Z) $\{(3)-(\overline{3})(2 m-3)-(\overline{1}, 2)\}$

+ Supp. $(\overline{3})$

$=3(\overline{3}) 2 D ;$

(2Z) $2\{(3)-(\overline{2}, 1)-(\overline{2}, 1)\}$

$+\{(2,1)-(\overline{2}, 1)(2 m-4)-(\overline{1}, 1,1) 2\}$

$+\operatorname{Supp}(\overline{2}, 1)$

$=2(\overline{2}, 1) 2 D ;$

(2Z) $3\{(3)-(\overline{1}, 2)-(\overline{3})(2 m-3)\}$

$+\{(1,2)-(\overline{1}, 2)(2 m-4)-(\overline{1}, 2)(2 m-4)\}$

+ Supp. $(\overline{1}, 2)$

$=(\overline{1}, 2) 2 D$;

(2Z) $2\{(2,1)-(\overline{1}, 1,1) 2-(\overline{2}, 1)(2 m-4)\}$

$+\{3(1,1,1)-(\overline{1}, 1,1)(2 m-5)-(\overline{1}, 1,1)(2 m-5)\}$

+ Supp. $(1,1,1)$

$=(\overline{1}, 1,1) 2 D$ :

(Z) $\quad\{(4)-(\overline{4})(2 m-4)-(\overline{1}, 3)\}$

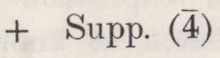

$=4(\overline{4}) 2 D$;

(Z) $2\{(4)-(\overline{3}, 1)-(\overline{2}, 2)\}$

$+\{(3,1)-(\overline{3}, 1)(2 m-5)-(\overline{1}, 1,2)\}$

+ Supp. $(\overline{3}, 1)$

$=3(\overline{3}, 1) 2 D$;

(Z) $3\{(4)-(\overline{2}, 2)-(3, \overline{1})\}$

$+\{2(2,2)-(\overline{2}, 2)(2 m-5)-(\overline{1}, 1,2)\}$

+ Supp. $(\overline{2}, 2)$

$=2(\overline{2}, 2) 2 D$;

(Z) $2\{(3,1)-(\overline{2}, 1,1) 2-(\overline{2}, 1,1) 2\}$

$+\{(2,1,1)-(\overline{2}, 1,1)(2 m-6)-(\overline{1}, 1,1,1) 3\}$

+ Supp. $(\overline{2}, 1,1)$

$=2(\overline{2}, 1,1) 2 D$

(Z) $4\{(4)-(\overline{1}, 3)-(\overline{4})(2 m-4)\}$

$+\{(1,3)-(\overline{1}, 3)(2 m-5)-(\overline{1}, 3)(2 m-5)\}$

+ Supp. $(\overline{1}, 3)$

$=(\overline{1}, 3) 2 D$; 
(Z) $3\{(3,1)-(\overline{1}, 1,2)-(\overline{3}, 1)(2 m-5)\}$

$+2\{2(2,2)-(\overline{1}, 1,2)-(\overline{2}, 2)(2 m-5)\}$

$+\{2(1,1,2)-(\overline{1}, 1,2)(2 m-6)-(\overline{1}, 1,2)(2 m-6)\}$

+ Supp. $(\overline{1}, 1,2)$ $=(\overline{1}, 1,2) 2 D$;

(Z) $2\{(2,1,1)-(\overline{1}, 1,1,1) 3-(\overline{2}, 1,1)(2 m-6)\}$

$+\{4(1,1,1,1)-(\overline{1}, 1,1,1)(2 m-7)-(\overline{1}, 1,1,1)(2 m-7)\}$

+ Supp. $(\overline{1}, 1,1,1)$ $=(\overline{1}, 1,1,1) 2 D$.

110. I content myself with giving the expressions of only the following supplements.

$$
\begin{aligned}
& \text { Supp. }(4 Z)(\overline{1})=m[2(\cdot)-(/)]+\kappa(\cdot) . \\
& \left.\begin{array}{rl}
\text { Supp. }(3 Z)(\overline{2})= & \frac{1}{2} n[2(:)-(\cdot /)]+\frac{1}{2} \kappa(\cdot /) . \\
\text { Supp. }(3 Z)(\overline{1}, 1)= & \left(\quad 2 m n-3 n^{2}-n+n \alpha\right)(:) \\
& +\left(2 m^{2}-4 m n-2 m+2 n+\left(m-\frac{1}{2}\right) \alpha\right)(\cdot /) \\
& +\left(-m^{2} \quad+m\right.
\end{array}\right)(/ /) .
\end{aligned}
$$

Supp. $(2 Z)(\overline{3})=-\frac{1}{4} m[2(\therefore)-(: /)]$

$$
\begin{aligned}
& +\frac{1}{4} n[2(\therefore)-(: /)+2(2(: /)-(\cdot / /))] \\
& +\frac{1}{2} \kappa(: /) .
\end{aligned}
$$

Supp. $(Z)(\overline{4})=a \kappa+b(2 \kappa+2 \iota)$, where $a, b$ are the representatives of the condition $Z$.

It may be added that we have in general

$$
\text { Supp. }(Z)(4 X)=a \text { Supp. }(4 X \cdot)+b \text { Supp. }(4 X /),
$$

where $(4 X)$ stands for any one of the symbols $(\overline{4}),(\overline{3}, 1) \ldots(\overline{1}, 1,1,1)$.

111. The expression of Supp. $(4 Z)(\overline{1})$ has been explained suprà, No. 108. That of Supp. $(3 Z)(2)$ may also be explained. $1^{\circ}$. The point-pairs of the system of conics $(3 Z)$, regarding each point-pair as a line, are a set of lines enveloping a curve; the class of this curve is equal to the number of the lines which pass through an arbitrary point, that is, as at first sight would appear, to the number of point-pairs in the system $(3 Z \cdot)$, or to $2(3 Z:)-(3 Z \cdot /)$ : it is, however, necessary to admit that the number of distinct lines, and therefore the elass of the curve, is one-half of this, or $=\frac{1}{2}[2(3 Z:)-(3 Z \cdot /)]$; which being so, the number of the point-pairs $(3 Z)$ which, regarded as lines, touch the given curve (of the order $m$ and class $n$ ) is $=\frac{1}{2} n[2(3 Z:)-(3 Z \cdot /)]$. The point of contact of any one of these lines with the given curve is (specially) a united point, and we have thus the term $\frac{1}{2} n[2(3 Z:)-(3 Z \cdot /)]$ of the Supplement. $2^{\circ}$. The number of the conics $(3 Z)$ which touch the given curve at a given cusp thereof, or, say, the conics $(3 Z)(2 \kappa 1)$, is $=\frac{1}{2}(3 Z \cdot /)$, and the cusp is in respect of each of these conics a united point; we have thus the remaining term $\frac{1}{2} \kappa(3 Z \cdot /)$ of the Supplement. 
Article Nos. 112 to 135.-Application to the Conics which satisfy five conditions of contact with a given Curve.

112. We have twelve equations, which I first present in what I call their original forms; viz. these are-

First equation :

$$
\{(\overline{5})-(\overline{5})(2 m-5)-(\overline{1}, 4)\}
$$

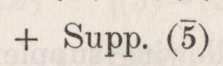

Second equation:

$$
\begin{aligned}
& 2\{(5)-(\overline{4}, 1)-(\overline{2}, 3)\} \\
+ & \{(4,1)-(\overline{4}, 1)(2 m-6)-(\overline{1}, 1,3)\} \\
+ & \text { Supp. }(\overline{4}, 1)
\end{aligned}
$$$$
=4(\overline{4}, 1) 2 D \text {. }
$$

Third equation:

$$
\begin{aligned}
& 3\{(\overline{5})-2(\overline{3}, 2)-2(\overline{3}, 2)\} \\
+ & \{(3,2)-(\overline{3}, 2)(2 m-6)-2(\overline{1}, 2,2)\} \\
+ & \text { Supp. }(\overline{3}, 2)
\end{aligned}
$$$$
=3(\overline{3}, 2) 2 D \text {. }
$$

Fourth equation:

$$
\begin{aligned}
& 2\{(4,1)-2(\overline{3}, 1,1)-(\overline{2}, 2,1)\} \\
& +\quad\{(3,1,1)-(\overline{3}, 1,1)(2 m-7)-(\overline{1}, 1,1,2)\} \\
& +\operatorname{Supp} .(\overline{3}, 1,1) \\
& =3(\overline{3}, 1,1) 2 D \text {. }
\end{aligned}
$$

Fifth equation:

$$
\begin{aligned}
& 4\{(5)-(\overline{2}, 3)-(\overline{4}, 1)\} \\
+ & \{(3,2)-(\overline{2}, 3)(2 m-6)-(\overline{1}, 1,3)\} \\
+ & \text { Supp. }(\overline{2}, 3)
\end{aligned}
$$$$
=2(\overline{2}, 3) 2 D \text {. }
$$

Sixth equation:

$$
\begin{aligned}
& 3\{(4,1)-2(\overline{2}, 2,1)-2,(\overline{3}, 1,1)\} \\
& +2\{(3,2)-(\overline{2}, 2,1)-(\overline{2}, 2,1)\} \\
& +\{2(2,2,1)-(\overline{2}, 2,1)(2 m-7)-2(\overline{1}, 1,1,2)\} \\
& + \text { Supp. }(\overline{2}, 2,1) \\
& =2(\overline{2}, 2,1) 2 D \text {. }
\end{aligned}
$$

Seventh equation:

$$
\begin{aligned}
& 2\{(3,1,1)-3(\overline{2}, 1,1,1)-3(\overline{2}, 1,1,1)\} \\
& +\{(2,1,1,1)-(\overline{2}, 1,1,1)(2 m-8)-4(\overline{1}, 1,1,1,1)\} \\
& + \text { Supp. }(\overline{2}, 1,1,1) \\
& =2(\overline{2}, 1,1,1) 2 D \text {. }
\end{aligned}
$$


Eighth equation:

$$
\begin{aligned}
& 5\{(5)-(\overline{1}, 4)-(\overline{5})(2 m-5)\} \\
+ & \{(4,1)-(\overline{1}, 4)(2 m-5)-(\overline{1}, 4)(2 m-5)\} \\
+ & \text { Supp. }(\overline{1}, 4)
\end{aligned}
$$

Ninth equation:

$$
\begin{aligned}
& 4\{(4,1)-(\overline{1}, 1,3)-(\overline{4}, 1)(2 m-6)\} \\
+ & 2\{(3,2)-(\overline{1}, 1,3)-(\overline{2}, 3)(2 m-6)\} \\
+ & \{2(3,1,1)-(\overline{1}, 1,3)(2 m-7)-(\overline{1}, 1,3)(2 m-7)\} \\
+ & \text { Supp. }(1,1,3)
\end{aligned}
$$

Tenth equation:

$$
\begin{aligned}
& 3\{(3,2)-2(\overline{1}, 2,2)-(\overline{3}, 2)(2 m-6)\} \\
+ & \{(2,2,1)-(\overline{1}, 2,2)(2 m-7)-(\overline{1}, 2,2)(2 m-7)\} \\
+ & \text { Supp. }(\overline{1}, 2,2)
\end{aligned}
$$

Eleventh equation:

$$
\begin{aligned}
& 3\{(3,1,1)-(\overline{1}, 1,1,2)-(\overline{3}, 1,1)(2 m-7)\} \\
& +2\{2(2,2,1)-2(\overline{1}, 1,1,2)-(\overline{2}, 2,1)(2 m-7)\} \\
& +\{3(2,1,1,1)-(\overline{1}, 1,1,2)(2 m-8)-(\overline{1}, 1,1,2)(2 m-8)\} \\
& + \text { Supp. }(\overline{1}, 1,1,2) \\
& =(\overline{1}, 1,1,2) 2 D \text {. }
\end{aligned}
$$

Twelfth equation:

$$
\begin{aligned}
& 2\{(2,1,1,1)-4(\overline{1}, 1,1,1,1)-(\overline{2}, 1,1,1)(2 m-8)\} \\
+ & \{5(1,1,1,1,1)-(\overline{1}, 1,1,1,1)(2 m-9)-(\overline{1}, 1,1,1,1)(2 m-9)\} \\
+ & \text { Supp. }(\overline{1}, 1,1,1,1) \\
= & =(\overline{1}, 1,1,1,1) 2 D .
\end{aligned}
$$

113. I alter the forms of these equations by substituting for $2 D$ its value $=n-2 m+2+\kappa$, and by writing for the expressions with $(\overline{1})$ their values,

$$
(\overline{1}, 4)=(\cdot 4)-5(\overline{5}), \& c .
$$

and except in the terms $\{$ Supp. $(\overline{5})-\kappa(\overline{5})\}$, \&c., by writing for $\kappa$ its value $-3 n+\alpha$. The resulting equations, if the Supplements were known, would serve to determine the values of $(5),(4,1)$, \&c.; but I assume instead that the last-mentioned expressions are known (First Memoir, No. 50), and use the equations to determine the Supplements, or, what comes to the same thing, the values of the terms in \{\} which contain these Supplements. We have thus the twelve reduced equations, with resulting values of the supplements.

C. VI. 
114. First equation:

$$
=0=
$$$$
+(\overline{5})(8 m+7 n-4 \alpha)
$$$$
-(\cdot 4)
$$

$$
\begin{array}{r}
-15 m-15 n+9 \alpha \\
-3 m+\alpha \\
8 m+7 n-4 \alpha \\
+10 m+8 n-6 \alpha
\end{array}
$$

(that is, we have

$$
\text { Supp. }(\overline{5})-\kappa(\overline{5})=-3 m+\alpha \text {, }
$$

and so in the subsequent cases, the equation gives the value of the term in $\{$ \} which contains the Supplement).

115. Second equation:

$$
\begin{aligned}
& +(4,1) \\
& +\{\text { Supp. }(\overline{4}, 1)-\kappa(\overline{4}, 1)\} \\
& +(\overline{4}, 1)(6 m+5 n-3 \alpha) \\
& -(\cdot 1,3) \\
& =0= \\
& -30 m-30 n+\alpha( \\
& -8 m^{2}-20 m n-8 n^{2}+104 m+104 n+\alpha(6 m+6 n-66) \\
& -6 m^{2}-3 m n+18 m+9 n+\alpha(3 m-9) \\
& +6 m^{2}+11 m n+5 n^{2}-36 m-30 n+\alpha(-3 m-3 n+18) \\
& +8 m^{2}+12 m n+3 n^{2}-56 m-53 n+\alpha(-6 m-3 n+39) \text {. }
\end{aligned}
$$

I stop for a moment to notice a very convenient verification of the term in \{\} ; putting therein $\alpha=3 n$, the term is

$$
-6 m^{2}-3 m n+18 m+9 n+(9 m n-27 n)
$$

and if in this we write $m=n=1, m^{2}=m n=n^{2}=2$, and when any higher terms enter $m^{3}=m^{2} n=m n^{2}=n^{3}=4, m^{4}=m^{3} n=m^{2} n^{2}=m n^{3}=n^{4}=8$, \&c., the value is $-12-6+18+9$ $+18-27,=0$, viz. we should always obtain a sum $=0$. The reason is that the term in question should always admit of being expressed in the form $p \delta+q \kappa+r \tau+s \iota$; the reduction to this form might be effected by the substitutions $m=\frac{1}{2}(m+n)+\frac{1}{6}(\kappa-\iota)$, $n=\frac{1}{2}(m+n)-\frac{1}{6}(\kappa-\iota), m^{2}=2 \cdot \frac{1}{2}(m+n)+2 \delta+3 \kappa, n^{2}=2 \cdot \frac{1}{2}(m+n)+2 \tau+3 \iota$, giving a result $=A(m+n)+$ terms in $(\delta, \kappa, \tau, \iota)$, where $A$ is a numerical coefficient calculable as above by simply writing $m=n=1, m^{2}=m n=n^{2}=2$, \&c., and which is $=0$ when the term is of the proper form $p \delta+q \kappa+r \tau+s i$. The complete reduction to the form in question is material in the sequel, but $\mathrm{I}$ advert to the point here only for the sake of the numerical verification.

116. Third equation :

$$
=0=
$$

$+\{$ Supp. $(\overline{3}, 2)-\kappa(\overline{3}, 2)\}$

$$
\text { - } 45 m-45 n+\alpha(
$$

$$
+120 m+120 n+\alpha(-4 m-4 n-78)+3 \alpha^{2}
$$

$+(\overline{3}, 2)(4 m+3 n-2 \alpha)$

$+15 m+\alpha(n-7)$

$-2(\cdot 2,2)$

$-36 m-27 n+\alpha(4 m+3 n+18)-2 \alpha^{2}$

$-54 m-48 n+\alpha($

$+40)-\alpha^{2}$

Verification is $15+3(1.2-7)=0$. 
117. Fourth equation:

$$
=0
$$

$$
2(4,1)
$$

$+\{$ Supp. $(\overline{3}, 1,1)-\kappa(\overline{3}, 1,1)\}$

$+(\overline{3}, 1,1)(4 m+3 n-2 \alpha)$

$-(\cdot 2,1,1)$

$$
\begin{array}{rr}
-16 m^{2}-40 m n-16 n^{2} \\
-\frac{3}{2} m^{3}-10 m^{2} n-10 m n^{2}-\frac{3}{2} n^{3}+\frac{109}{2} m^{2}+116 m n+\frac{109}{2} n^{2} \\
-\frac{1}{2} m^{3}+\frac{1}{2} m^{2} n+2 m n^{2}+\frac{23}{2} m^{2}+\frac{11}{2} m n-7 n^{2} \\
2 m^{3}+\frac{19}{2} m^{2} n+8 m n^{2}+\frac{3}{2} n^{3}-26 m^{2}-\frac{91}{2} m n-\frac{39}{2} n^{2} \\
-24 m^{2}-36 m n-12 n^{2}
\end{array}
$$

(1) $\quad+208 m+208 n+\alpha(\quad+12 m+12 n-132)$

(2) $-434 m-434 n+\alpha\left(\frac{3}{2} m^{2}+6 m n+\frac{3}{2} n^{2}-\frac{69}{2} m-\frac{69}{2} n+291\right)-\frac{9}{2} \alpha^{2}$

(3) $\quad-50 m-23 n+\alpha\left(\frac{1}{2} m^{2} \quad-\frac{19}{2} m-\frac{1}{2} n+33\right)$

(4) $+108 m+81 n+\alpha\left(-m^{2}-4 m n-n^{2}+7 m+\frac{17}{2} n-54\right)+3 \alpha^{2}$

(5) $\quad+168 m+168 n+\alpha\left(-m^{2}-2 m n-\frac{1}{2} n^{2}+25 m+\frac{29}{2} n-138\right)+\frac{3}{2} \alpha^{2}$.

Verification is $\left(-\frac{1}{2}+\frac{1}{2}+2\right) 4+\left(\frac{23}{2}+\frac{11}{2}-7\right) 2-50-23+3\left(\frac{1}{2} \cdot 4-\left(\frac{19}{2}+\frac{1}{2}\right) 2+33\right)=0$.

118. Fifth equation:

$$
=0=
$$

\section{$4(5)$}

$+(3,2)$

$+\{\operatorname{Supp},(\overline{2}, 3)-\kappa(\overline{2}, 3)\}$

$+(\overline{2}, 3)(2 m+n-\alpha)$

$-(\cdot 1,3)$

$$
\begin{aligned}
&-60 m-60 n+\alpha(36) \\
&+ 120 m+120 n+\alpha(-4 m-4 n-78)+3 x^{2} \\
& n^{2}+8 m-\quad n+\alpha(-3) \\
&-8 m^{2}-12 m n-4 n^{2}-12 m-6 n+\alpha(10 m+7 n+6)-3 \alpha^{2} \\
&+8 m^{2}+12 m n+3 n^{2}-56 m-53 n+\alpha(-6 m-3 n+39) .
\end{aligned}
$$

Verification is $2+8-1+3(-3)=0$.

119. Sixth equation:

$$
=0
$$

$\begin{aligned} & 3(4,1) \\ + & 2(3,2) \\ + & 2(2,2,1) \\ + & \{\operatorname{Supp} .(\overline{2}, 2,1)-\kappa(\overline{2}, 2,1)\} \\ + & (\overline{2}, 2,1)(2 m+n-\alpha) \\ - & 2(\cdot 2,1,1)\end{aligned}$

$$
\begin{array}{r}
-24 m^{2}-60 m n-24 n^{2}+312 m+312 n \\
+240 m+240 n \\
+48 m^{2}+108 m n+48 n^{2}-936 m-936 n \\
+12 m^{2}+6 m n-6 n^{2}-60 m-6 n \\
+12 m^{2}+18 m n+6 n^{2}+108 m+54 n \\
-48 m^{2}-72 m n-24 n^{2}+336 m+336 n
\end{array}
$$




$$
\begin{array}{lll}
\text { (1) } & +\alpha( & +18 m+18 n-198) \\
\text { (2) } & +\alpha( & -8 m-8 n-156)+\alpha^{2}( \\
\text { (3) } & +\alpha( & -16 m-16 n+654)+\alpha^{2}(m+n-24) \\
\text { (4) } & +\alpha( & -8 m-2 n+30) \\
\text { (5) } & +\alpha\left(2 m^{2}+3 m n+n^{2}-36 m-21 n-54\right)+\alpha^{2}(-m-n+15) \\
\text { (6) } & +\alpha\left(-2 m^{2}-4 m n-n^{2}+50 m+29 n-276\right)+\alpha^{2}( & +3) .
\end{array}
$$

Verification is $(12+6-6) 2-60-6+3(4-(8+2) 2+30)=0$

120. Seventh equation:

$$
=0=
$$

$$
2(3,1,1)
$$

$+(2,1,1,1)$

$+\{\operatorname{Supp} .(\overline{2}, 1,1,1)-\kappa(\overline{2}, 1,1,1)\}$

$+(\overline{2}, 1,1,1)(2 m+n-a)$

$-(\cdot 1,1,1,1)$

$$
\begin{aligned}
& \text { (1) }+\alpha\left(\quad 3 m^{2}+12 m n+3 n^{2}-69 m-69 n+582\right)+\alpha^{2}( \\
& \text { (2) } \quad+\alpha\left(\frac{1}{6} m^{3}+m^{2} n+m n^{2}+\frac{1}{6} n^{3}-\frac{15}{2} m^{2}-26 m n-\frac{15}{2} n^{2}+\frac{358}{3} m+\frac{358}{3} n-960\right)+\alpha^{2}\left(-\frac{3}{2} m-\frac{3}{2} n+28\right) \\
& \text { (3) } \quad+\alpha\left(-m^{2}-\frac{3}{2} m n+\frac{1}{2} n^{2}+19 m+\frac{5}{2} n-54\right) \\
& \text { (4) } \quad+\alpha\left(-\frac{1}{6} m^{3}-m^{2} n-m n^{2}-\frac{1}{6} n^{3}-\frac{1}{2} m^{2}+\frac{7}{2} m n+n^{2}+\frac{50}{3} m+\frac{50}{3} n+75\right)+\alpha^{2}\left(\frac{3}{2} m+\frac{3}{2} n-\frac{29}{2}\right) \\
& \text { (5) }+\alpha( \\
& \left.6 m^{2}+12 m n+3 n^{2}-86 m-55 n+357\right)+\alpha^{2}(
\end{aligned}
$$

Verification is

$$
\left(\frac{1}{2}+1\right) 8+\left(-\frac{7}{2}-5-\frac{3}{2}\right) 4+\left(-20-5+\frac{29}{2}\right) 2+80+26+3\left(\left(-1-\frac{3}{2}+\frac{1}{2}\right) 4+\left(19+\frac{5}{2}\right) 2-54\right)=0 .
$$


121. Eighth equation :

$$
=0
$$

$$
\begin{aligned}
&- 75 m-75 n+\alpha( \\
&-8 m^{2}-20 m n-8 n^{2}+104 m+104 n+\alpha(6 m+6 n-66) \\
&+1 m-4 n+\alpha( \\
& 10 m n+8 n^{2}-\frac{50}{3} m-\frac{40}{3} n+\alpha(-6 n+10) \\
& 8 m^{2}+10 m n .-\frac{40}{3} m-\frac{50}{3} n+\alpha(-6 m+10) \\
&+5 n .
\end{aligned}
$$$$
+(4,1)
$$$$
+\left(-n+\frac{5}{3}\right)(\cdot 4)
$$$$
+\left(-m+\frac{5}{3}\right)(/ 4)
$$$$
+5 n(\overline{5})
$$

Verification is $1-4+3.1=0$.

122. Ninth equation:

$$
=0=
$$

$$
\begin{aligned}
& 4(4,1) \\
+ & 2(3,2) \\
+ & 2(3,1,1) \\
+ & \left\{\begin{array}{l}
\text { Supp. }(\overline{1}, 1,3)-\kappa(\overline{1}, 1,3) \\
-(m-2)(2(\cdot 1,3)-(/ 1,3))
\end{array}\right\} \\
+ & (-n+2)(\cdot 1,3) \\
+ & (-m+2)(/ 1,3) \\
+ & n(4(\overline{4}, 1)+2(\overline{2}, 3))
\end{aligned}
$$$$
-32 m^{2}-80 m n-32 n^{2}(1)
$$$$
-3 m^{3}-20 m^{2} n-20 m n^{2}-3 n^{3}+109 m^{2}+232 m n+109 n^{2}
$$$$
-2 m^{2}+12 m n+2 n^{2}
$$$$
+8 m^{2} n+12 m n^{2}+3 n^{3}-16 m^{2}-80 m n-59 n^{2}
$$$$
+3 m^{3}+12 m^{2} n+8 m n^{2} \quad-59 m^{2}-80 m n-16 n^{2} \text { (6) }
$$$$
\text { - } 4 m n-4 n^{2}
$$

$$
\begin{array}{lrr}
\text { (1) } & +416 m+416 n+\alpha( & +24 m+24 n-264) \\
\text { (2) } & +240 m+240 n+\alpha( & -8 m-8 n-156)+6 \alpha^{2} \\
\text { (3) } & -868 m-868 n+\alpha\left(3 m^{2}+12 m n+3 n^{2}-69 m-69 n+582\right)-9 \alpha^{2} \\
\text { (4) } & -6 m+30 n+\alpha( & -4 m-10 n-6)+3 \alpha^{2} \\
\text { (5) } & +112 m+106 n+\alpha( & \left.-6 m n-3 n^{2}+12 m+45 n-78\right) \\
\text { (6) } & +106 m+112 n+\alpha\left(-3 m^{2}-6 m n\right. & +45 m+12 n-78) \\
\text { (7) } & -36 n+\alpha( &
\end{array}
$$

Verification is $(-2+12+2) 2-6+30+3((-4-10) 2-6)+3 \cdot 9 \cdot 2=0$ 
123. Tenth equation:

$$
=0=
$$

$3(3,2)$

$+(2,2,1)$

$+\left\{\begin{array}{l}\text { Supp. }(\overline{1}, 2,2)-\kappa(\overline{1}, 2,2) \\ -(m-2)(2(\cdot 2,2)-(/ 2,2))\end{array}\right\}$

$+(-n+2)(\cdot 2,2)$

$+(-m+2)(/ 2,2)$

$+3 n(\overline{3}, 2)$

$$
\begin{array}{r}
+360 m+360 n \\
24 m^{2}+54 m n+24 n^{2}-468 m-468 n \\
+6 m+33 n \\
-27 m n-24 n^{2}+54 m+48 n \\
-24 m^{2}-27 m n \\
+48 m+54 n \\
-27 n
\end{array}
$$

$$
\begin{aligned}
& \text { (1) }+\alpha(-12 m-12 n-234)+\alpha^{2}(+9) \\
& \text { (2) }+\alpha(-8 m-8 n+327)+\alpha^{2}\left(\frac{1}{2} m+\frac{1}{2} n-12\right) \\
& \text { (3) }+\alpha(-3 n-13)+\alpha^{2}(+1) \\
& \text { (4) }+\alpha(+20 n-40)+\alpha^{2}\left(-\frac{1}{2} n+1\right) \\
& \text { (5) }+\alpha(20 m-40)+\alpha^{2}\left(\frac{1}{2} m+1\right) \\
& \text { (6) }+\alpha(\quad+3 n+
\end{aligned}
$$

Verification is $+33+3(-3.2-13)+9.2=0$

124. Eleventh equation :

$$
=0=
$$

\begin{tabular}{r|c} 
& $3(3,1,1)$ \\
+ & $4(2,2,1)$ \\
+ & $3(2,1,1,1)$ \\
+ & $\left\{\begin{array}{c}\text { Supp. }(\overline{1}, 1,1,2)-\kappa(\overline{1}, 1,1,2) \\
-\left(m-\frac{7}{3}\right)(2(\cdot 1,1,2)-(/ 1,1,2)\end{array}\right\}$ \\
+ & $\left(-n+\frac{7}{3}\right)(\cdot 1,1,2)$ \\
+ & $\left(-m+\frac{7}{3}\right)(/ 1,1,2)$ \\
+ & $n\left(3(\overline{3}, 1,1)+2\left(\frac{3}{2}\right)\right.$
\end{tabular}


(1) $\quad+\frac{327}{2} m^{2}+348 m n+\frac{327}{2} n^{2}-1302 m-1302 n+\alpha($

(2) $\quad+96 m^{2}+216 m n+96 n^{2}-1872 m-1872 n+\alpha($

(3) $\quad-522 m^{2}-1044 m n-522 n^{2}+3960 m+3960 n+\alpha\left(\frac{1}{2} m^{3}+3 m^{2} n+3 m n^{2}+\frac{1}{2} n^{3}\right.$

(4) $\quad+\frac{21}{2} m^{2}-\frac{33}{2} m n+18 n^{2}-2 m-191 n+\alpha($

(5) $+56 m^{2}+252 m n+196 n^{2}-392 m-392 n+\alpha\left(\quad-m^{2} n-2 m n^{2}-\frac{1}{2} n^{3}\right.$

(6) $+196 m^{2}+252 m n+56 n^{2}-392 m-392 n+\alpha\left(-\frac{1}{2} m^{3}-2 m^{2} n-m n^{2}\right.$

(7)

$$
-\frac{15}{2} m n-\frac{15}{2} n^{2}+189 n+\alpha(
$$

(1) $\left.\quad \frac{9}{2} m^{2}+18 m n+\frac{9}{2} n^{2}-\frac{207}{2} m-\frac{207}{2} n+873\right)+\alpha^{2}\left(-\frac{27}{2}\right)$

(2) $\quad-32 m-32 n+1308)+\alpha^{2}(2 m+2 n-48)$

(3) $\left.\quad-\frac{45}{2} m^{2}-78 m n-\frac{45}{2} n^{2}+358 m+358 n-2880\right)+\alpha^{2}\left(-\frac{9}{2} m-\frac{9}{2} n+84\right)$

(4) $\left.-\frac{4}{3} m n-2 n^{2}+\frac{23}{3} m+\frac{253}{6} n+55\right)+\alpha^{2}\left(m+n-\frac{31}{2}\right)$

(5) $\left.\quad+\frac{7}{3} m^{2}+\frac{89}{3} m n+\frac{47}{3} n^{2}-\frac{175}{3} m-\frac{1031}{6} n+322\right)+\alpha^{2}\left(\frac{3}{2} m-\frac{7}{2}\right)$

(6) $\left.\quad+\frac{47}{3} m^{2}+\frac{89}{3} m n+\frac{7}{3} n^{2}-\frac{1031}{6} m-\frac{175}{3} n+322\right)+\alpha^{2}\left(+\frac{3}{2} n-\frac{7}{2}\right)$

(7) $2 m n+2 n^{2} \quad \frac{69}{2} n \quad$ ).

Verification is $4\left(-\frac{3}{2}-\frac{3}{2}-6-3\right)+2\left(\frac{21}{2}-\frac{33}{2}+18\right)-2-191$

$$
+3\left(\left(-\frac{4}{3}-2\right) 4+\left(\frac{23}{3}+\frac{25}{6}\right) 2+55\right)+9\left((1+1) 4-\frac{31}{2} \cdot 2\right)=0 .
$$

125. Twelfth equation :

$$
=0=
$$

$2(\overline{2}, 1,1,1)$

$+5(1,1,1,1,1)$

$+\left\{\begin{array}{r}\operatorname{Supp} \cdot(\overline{1}, 1,1,1,1)-\kappa(\overline{1}, 1,1,1,1) \\ -\left(m-\frac{8}{3}\right)(2(\cdot 1,1,1,1)-(/ 1,1,1,1))\end{array}\right\}$

$\frac{1}{24} m^{5}+\frac{5}{12} m^{4} n+\frac{5}{3} m^{3} n^{2}+\frac{5}{3} m^{2} n^{3}+\frac{5}{12} m n^{4}+\frac{1}{24} n^{5}$

$+\left(-n+\frac{8}{3}\right)(\cdot 1,1,1,1)$

$+\left(-m+\frac{8}{3}\right)(/ 1,1,1,1)$

$-\frac{1}{12} m^{4} n-\frac{2}{3} m^{3} n^{2}-m^{2} n^{3}-\frac{1}{3} m n^{4}-\frac{1}{24} n^{5}$

$-\frac{1}{24} m^{5}-\frac{1}{3} m^{4} n-m^{3} n^{2}-\frac{2}{3} m^{2} n^{3}-\frac{1}{12} m n^{4}$

$+2 n(\overline{2}, 1,1,1)$ 
(1)

$$
+12 m^{3}+60 m^{2} n+60 m n^{2}+12 n^{3}
$$

(2) $\quad-\frac{5}{12} m^{4}-\frac{25}{6} m^{3} n-10 m^{2} n^{2}-\frac{25}{6} m n^{3}-\frac{5}{12} n^{4}-\frac{565}{24} m^{3}-\frac{1045}{12} m^{2} n-1045 \frac{5}{12} m n^{2}-\frac{565}{24} n^{3}$

.

- $\frac{1}{6} m^{4}-\frac{4}{3} m^{3} n-\frac{10}{3} m^{2} n^{2}-3 m n^{3}-\frac{1}{2} n^{4}+4 m^{3}+\frac{28}{3} m^{2} n+\frac{61}{3} m n^{2}+9 n^{3}$

(4) $\quad+\frac{2}{9} m^{4}+\frac{41}{18} m^{3} n+\frac{17}{3} m^{2} n^{2}+\frac{26}{9} m n^{3}+\frac{13}{36} n^{4}-\frac{4}{3} m^{3}+\frac{85}{12} m^{2} n+\frac{47}{3} m n^{2}+\frac{213}{24} n^{3}$

(5) $\quad+\frac{13}{36} m^{4}+\frac{26}{9} m^{3} n+\frac{17}{3} m^{2} n^{2}+\frac{41}{18} m n^{3}+\frac{2}{9} n^{4}+\frac{213}{24} m^{3}+\frac{47}{3} m^{2} n+\frac{85}{12} m n^{2}-\frac{4}{3} n^{3}$

$$
\frac{1}{3} m^{3} n+2 m^{2} n^{2}+2 m n^{3}+\frac{1}{3} n^{4} \quad-\quad 5 m^{2} n-16 m n^{2}-5 n^{3}
$$

(1) $\quad-348 m^{2}-696 m n-348 n^{2}+2640 m+2640 n+\alpha\left(\frac{1}{3} m^{3}+2 m^{2} n+2 m n^{2}+\frac{1}{3} n^{3}\right.$

(2) $\quad+\frac{6335}{12} m^{2}+\frac{2965}{3} m n+\frac{6335}{12} n^{2}-3159 m-3159 n+\alpha\left(-\frac{5}{4} m^{3}-\frac{15}{2} m^{2} n-\frac{15}{2} m n^{2}-\frac{5}{4} n^{3}\right.$

(3) $\quad-\frac{27}{2} m^{2}-14 m n-\frac{229}{6} n^{2}-\frac{13}{3} m+\frac{437}{3} n+\alpha\left(\frac{1}{6} m^{3}+m^{2} n+m n^{2}+\frac{1}{6} n^{3}\right.$

(4)

$$
\begin{aligned}
& -\frac{362}{9} m^{2}-\frac{302}{2} m n-\frac{4543}{36} n^{2}+\frac{764}{3} m+\frac{806}{3} n+\alpha( \\
& \frac{3}{2} m^{2} n+3 m n^{2}+\frac{3}{4} n^{3} \\
& -\frac{4543}{36} m^{2}-\frac{303}{2} m n-\frac{362}{9} n^{2}+\frac{806}{3} m+\frac{764}{3} n+\alpha\left(\frac{3}{4} m^{3}+3 m^{2} n+\frac{2}{3} m n^{2}\right.
\end{aligned}
$$

(1) $\left.\quad-15 m^{2}-52 m n-15 n^{2}+\frac{716}{3} m+\frac{716}{3} n-1920\right)+\alpha^{2}(-3 m-3 n+56)$

(2)

$\left.+\frac{145}{4} m^{2}+115 m n+\frac{145}{4} n^{2}-\frac{1685}{4} m-\frac{1685}{4} n+2430\right)+\alpha^{2}\left(\frac{45}{8} m+\frac{45}{8} n-75\right)$

(3)

$-\frac{3}{2} m^{2}-m n+\frac{3}{2} n^{2}-\frac{2}{3} m-\frac{89}{3} n-$

$34)+\alpha^{2}\left(-\frac{3}{2} m-\frac{3}{2} n+13\right)$

- $4 m^{2}-\frac{59}{2} m n-\frac{63}{4} n^{2}+\frac{172}{3} m+\frac{1511}{12} n-$

$238)+\alpha^{2}($

$\left.-\frac{9}{8} n+3\right)$

$-\frac{63}{4} m^{2}-\frac{5}{2}$

$$
3 m n-3 n^{2}
$$

$$
+29 n
$$

Verification is

$$
\begin{aligned}
& \left(-\frac{1}{6}-\frac{4}{3}-\frac{10}{3}-3-\frac{1}{2}\right) 8+\left(4+\frac{28}{3}+\frac{61}{3}+9\right) 4+\left(-\frac{27}{2}-14-\frac{229}{6}\right) 2+\left(-\frac{13}{3}+\frac{437}{3}\right) \\
& \quad+3\left(\left(\frac{1}{6}+1+1+\frac{1}{6}\right) 8+\left(-\frac{3}{2}-1+\frac{3}{2}\right) 4+\left(-\frac{2}{3}-\frac{89}{3}\right) 2-34\right)+9\left(\left(-\frac{3}{2}-\frac{3}{2}\right) 4+13.2\right)=0 .
\end{aligned}
$$

126. It will be observed that in the eighth and following equations, viz. those

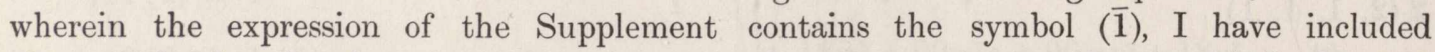
along with the Supplement within the \{\} , the terms $-\left(m-\frac{5}{3}\right)\{2(\cdot 4)-(/ 4)\}$, \&c., viz. these are $-\left(m-\frac{5}{3}\right)$ into number of point-pairs (4), \&c.: this is for convenience only; it simplifies the calculation, both from the symmetrical form under which the remaining terms present themselves in the several equations, and because the expressions of the terms in question, (these terms being mere multiples of a number of point-pairs) are by Zeuthen's theory known in terms of the Capitals. It is to be noticed that for any equation, to find the system to which the Capitals belong, we diminish by unity the barred number and then remove the bar; thus for the seventh equation, where we have Supp. $(\overline{2}, 1,1,1)$, the Capitals belong to the system $(1,1,1,1)$. 
127. Referring to Nos. 41 to 47 of the First Memoir, for convenience I collect the capitals which belong to a single curve, giving the values in terms of $m, n, \alpha$ as follows.

$$
(1,1,1,1)
$$

(1) $A=\frac{1}{2} \delta(\delta-1)$

$$
=\frac{1}{8} m^{4}
$$$$
-\frac{1}{4} m^{3}+2 m^{2} n
$$$$
-\frac{1}{8} m^{2}-2 m n+8 n^{2}+\frac{1}{4} m-2 n
$$

(2) $B=\delta(n-4)(m-4)=\frac{1}{2} m^{3} n$

$$
+\alpha\left(-\frac{2}{3} m^{2}+\frac{3}{4} m-6 n+\frac{3}{4}\right)+\frac{9}{8} \alpha^{2}
$$

(4) $C=\tau \cdot \frac{1}{2}(m-4)(m-5)=$

(3) $D=\iota \cdot \frac{1}{2}(m-3)(m-4)=$

$(2,1,1)$

(3) $E=\delta(n-4)$

(3) $\quad F=2 \delta(m-3)$

$=\quad m^{3}$

(6) $G=2 \tau(m-4)=$

(2) $D\left[=\iota \cdot \frac{1}{2}(m-3)(m-4)\right.$ suprà $]$;

(1) $H=\delta \kappa$ $=$

(2) $I=\kappa(n-3)(m-4)=$

(5) $J=\iota(m-3)$

$=$

$$
=
$$

$-2 m^{3}-\frac{5}{2} m^{2} n+4 m n^{2}+10 m^{2}-14 m n-16 n^{2}-8 m+64 n$ $+\alpha\left(-\frac{3}{2} m n+6 m+6 n-24\right) ;$ $\frac{1}{4} m^{2} n^{2}+2 m^{3}-\frac{1}{4} m^{2} n-\frac{9}{4} m n^{2}-18 m^{2}+\frac{9}{4} m n+5 n^{2}+40 n-5 n$

$$
+\alpha\left(-\frac{3}{4} m^{2} \quad+\frac{27}{4} m-15\right) \text {; }
$$

$-\frac{3}{2} m^{3}$

$+\frac{21}{2} m^{2}$

$-18 m$

$+a\left(\frac{1}{2} m^{2}\right.$

$-\frac{7}{2} m$

$+6)$ $-\frac{3}{2} m^{2} n$

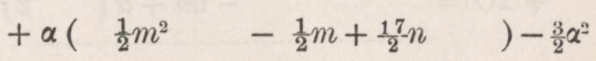$$
-3 m n^{2}+9 m n+12 n^{2}
$$$$
+a(\quad m n-3 m-4 n+12) \text {; }
$$

$-3 m^{2}$

$(2,2)$

(9) $K=\tau$

(3) $L=\kappa(n-3)$

(1) $M=\frac{1}{2} \kappa(\kappa-1)$

(2) $N=\imath$

(1) $0=\kappa$

$$
\begin{array}{lrrr}
= & \frac{1}{2} n^{2}+4 m-\frac{1}{2} n+\alpha( & \left.-\frac{3}{2}\right) ; \\
= & -3 n^{2} & +9 n+\alpha( & n-3) ; \\
= & \frac{9}{2} n^{2} & +\frac{3}{2} n+\alpha\left(-3 n-\frac{1}{2}\right)+\frac{1}{2} \alpha^{2} ; \\
= & -3 m+\alpha( & 1) ; \\
= & -3 n+\alpha( & 1) .
\end{array}
$$

c. VI. 
$(3,1)$
(2) $P=2 \delta$
$=m^{2}$
$-m+8 n+\alpha(-3)$;
(2) $Q=2 \tau$
$=n^{2}+8 m-n+\alpha(-3)$;
(5) $J[=\iota(m-3)$ suprà $]$;
(4) $R=\kappa(m-3)$
$=-3 m n+9 n+\alpha(m-3)$.

(4)

(4) $N[=\iota$ suprà $]$;

(2) $O[=\kappa$ suprà $]$.

128. I make the following calculations, serving to express in terms of Zeuthen's Capitals, the terms in \{ $\}$ contained in the twelve equations respectively.

$$
\begin{aligned}
& N=-3 m+\alpha \\
& \overline{-3 m+\alpha} \text { (first equation). } \\
& 2 J=-6 m^{2}+18 m+\alpha(2 m-6) \\
& +R=-3 m n+9 n \quad+\alpha(m-3) \\
& -6 m^{2}-3 m n+18 m+9 n+\alpha(3 m-9) \text { (second equation). } \\
& 6 K=3 n^{2}+24 m-3 n+\alpha(-9) \\
& +L=-3 n^{2} \quad+9 n+\alpha(n-3) \\
& +3 N=\quad-9 m+\alpha(3) \\
& +20=\quad-6 n+\alpha(2) \\
& 15 m+\alpha(n-7) \text { (third equation). } \\
& E=\quad \frac{1}{2} m^{2} n \quad-2 m^{2}-\frac{1}{2} m n+4 n^{2}+2 m-16 n+\alpha\left(\quad-\frac{3}{2} n+6\right) \\
& +F=m^{3} \quad-4 m^{2}+8 m n \quad+3 m-24 n+\alpha(-3 m+9) \\
& +2 G=\quad 2 m n^{2}+16 m^{2}-2 m n-8 n^{2}-64 m+8 n+\alpha(-6 m+24) \\
& +D=-\frac{3}{2} m^{3}+\frac{21}{2} m^{2} \quad-18 m+\alpha\left(\frac{1}{2} m^{2}-\frac{7}{2} m+6\right) \\
& +3 J=\quad-9 m^{2} \quad+27 m \quad+\alpha(\quad 3 m-9) \\
& +J^{\prime}=\quad-3 n^{2}+\quad 9 n+\alpha(n-3) \\
& -\frac{1}{2} m^{3}+\frac{1}{2} m^{2} n+2 m n^{2}+\frac{23}{2} m^{2}+\frac{11}{2} m n-7 n^{2}-50 m-23 n+\alpha\left(\frac{1}{2} m^{2}-\frac{19}{2} m-\frac{1}{2} n+33\right)
\end{aligned}
$$

(fourth equation) 


$$
Q=\frac{n^{2}+8 m-n-3 \alpha}{n^{2}+8 m-n-3 \alpha} \text { (fifth equation) }
$$

$$
\begin{aligned}
& 3 G=3 m n^{2}+24 m^{2}-3 m n-12 n^{2}-96 m+12 n+\alpha(-9 m+36) \\
& +I=-3 m n^{2} \quad+9 m n+12 n^{2} \quad-36 n+\alpha(m n-3 m-4 n+12) \\
& +4 J=-12 m^{2}+36 m+\alpha(4 m-12) \\
& +2 J^{\prime}=\quad-6 n^{2} \quad+18 n+\alpha(2 n-6) \\
& 12 m^{2}+6 m n-6 n^{2}-60 m-6 n+\alpha(m n-8 m-2 n+30)
\end{aligned}
$$

(sixth equation).

$$
\begin{aligned}
& B=\frac{1}{3} m^{3} n \quad-2 m^{3}-\frac{5}{2} m^{2} n+4 m n^{2} \quad+10 m^{2}-14 m n-16 n^{2}-8 m+64 n \\
& +4 C=\quad m^{2} n^{2}+8 m^{3}-m^{2} n-9 m n^{2} \quad-72 m^{2}+9 m n+20 n^{2}+160 m-20 n \quad \text { (2) } \\
& +4 D=-6 m^{3}+42 m^{2} \quad-72 m \\
& +D^{\prime}=\quad-\frac{3}{2} n^{3} \quad+\frac{21}{2} n^{2} \quad-18 n \quad \text { (4) } \\
& \frac{1}{2} m^{3} n+m^{2} n^{2} \quad-\frac{7}{2} m^{2} n-5 m n^{2}-\frac{3}{2} n^{3}-20 m^{2}-5 m n+\frac{29}{2} n^{2}+80 m+26 n \\
& \text { (1) } \quad+\alpha\left(-\frac{3}{2} m n+6 m+6 n-24\right) \\
& \text { (2) }+\alpha\left(-3 m^{2}+27 m-60\right) \\
& \text { (3) }+\alpha\left(2 m^{2}-14 m+24\right) \\
& \text { (4) } \quad+\alpha\left(\frac{1}{2} n^{2} \quad-\frac{7}{2} n+6\right) \\
& \text { (5) }+\alpha\left(-m^{2}-\frac{3}{2} m n+\frac{1}{2} n^{2}+19 m+\frac{5}{2} n-54\right) \text { (seventh equation). } \\
& -\frac{1}{3} N=m \quad-\frac{1}{3} \alpha \\
& +\frac{4}{3} O=-4 n+\frac{4}{3} \alpha \\
& m-4 n+\alpha \text { (eighth equation). }
\end{aligned}
$$

$$
\begin{aligned}
(\overline{2}, 3) & =-4 m-4 n-6+3 \alpha \\
+2(\overline{4}, 1) & =\frac{2 m+2 n-12}{-2 m-2 n-18+3 x} \text { (used infrà) }
\end{aligned}
$$

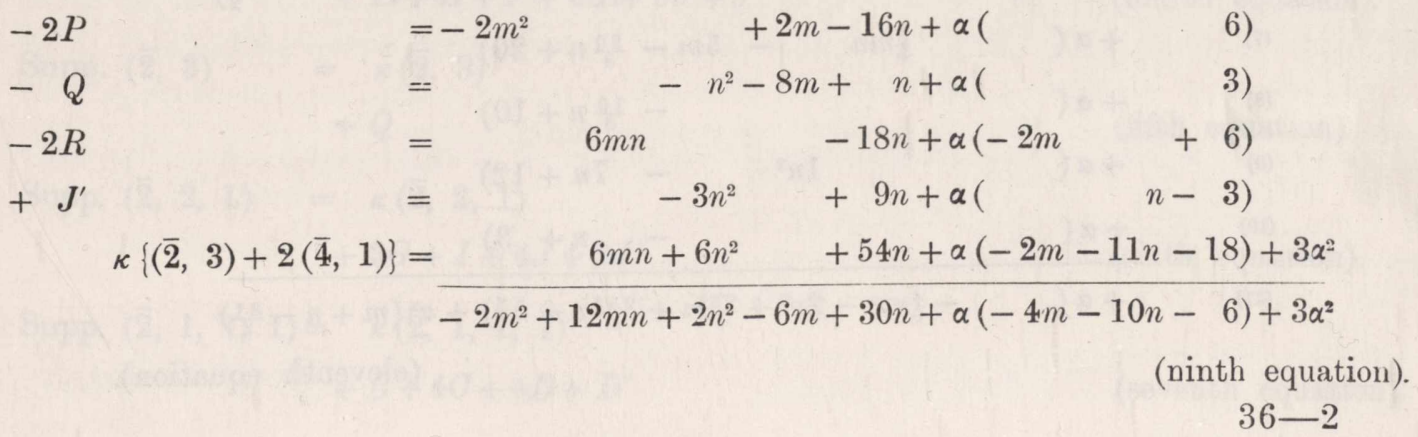




$$
\begin{array}{rll}
3 L & =-9 n^{2} & +27 n+\alpha(3 n-9) \\
+2 M & =9 n^{2} \quad+3 n+\alpha(-6 n-1)+\alpha^{2} \\
-2 N & = & +6 m+\alpha(-2) \\
-O & = & \frac{3 n+\alpha(-1)}{6 m+33 n+\alpha(-3 n-13)+\alpha^{2}} \text { (tenth equation). }
\end{array}
$$

$$
\begin{aligned}
& \kappa(\overline{2}, 2,1)= \\
& -18 m n-18 n^{2} \quad-162 n \\
& -\frac{1}{3} D=\frac{1}{2} m^{3} \\
& -\frac{7}{2} m^{2} \\
& +6 m \\
& -2 E \quad-\quad-m^{2} n \\
& +4 m^{2}+1 m n-8 n^{2}-4 m+32 n \\
& -2 F \quad=-2 m^{3} \\
& +8 m^{2}-16 m n \\
& -6 m+48 n \\
& -G \quad= \\
& -m n^{2} \\
& -8 m^{2}+1 m n+4 n^{2}+32 m-4 n \\
& +\frac{1}{3} H=-\frac{1}{2} m^{2} n \\
& +\frac{1}{2} m n-4 n^{2} \\
& 3 n^{2}-9 n
\end{aligned}
$$

$$
\begin{aligned}
& \text { (1) } \quad+\alpha\left(\quad-3 m n-3 n^{2}+6 m+51 n+54\right)+\alpha^{2}(m+n-15) \\
& \text { (2) } \quad+\alpha\left(-\frac{1}{6} m^{2}\right. \\
& +\frac{7}{6} m \\
& \text { - 2) } \\
& \text { (3) } \quad+\alpha( \\
& 3 n-12) \\
& \text { (4) } \quad+\alpha( \\
& 6 m \\
& -18) \\
& \text { (5) }+\alpha( \\
& 3 m \\
& -12) \\
& \text { (6) } \quad+\alpha\left(\frac{1}{6} m^{2}\right. \\
& \left.-\frac{1}{6} m+\frac{17}{6} n \quad\right)+\alpha^{2}( \\
& +\alpha( \\
& \frac{5}{3} m n \\
& \text { - } 5 m-\frac{20}{3} n+20 \text { ) } \\
& \text { (8) } \quad+a( \\
& \left.-\frac{10}{3} n+10\right) \\
& \text { (9) }+\alpha( \\
& 1 n^{2} \\
& \text { - } 7 n+12) \\
& +\alpha(-n+3) \\
& +\alpha\left(\quad-\frac{4}{3} m n-2 n^{2}+\frac{23}{3} m+\frac{253}{6} n+55\right)+\alpha^{2}\left(m+n-\frac{31}{2}\right)
\end{aligned}
$$

(eleventh equation). 
$\kappa(\overline{2}, 1,1,1)=\quad-\frac{1}{2} m^{3} n-3 m^{2} n^{2}-3 m n^{3}-\frac{1}{2} n^{4} \quad+\frac{15}{2} m^{2} n+24 m n^{2}+\frac{15}{2} n^{3} \quad-37 m n-37 n^{2}$

$\begin{array}{lllll}-\frac{4}{3} A & =-\frac{1}{6} m^{4} & +\frac{1}{3} m^{3}-\frac{3}{3} m^{2} n & +\frac{1}{6} m^{2}+\frac{8}{3} m n-\frac{32}{3} n^{2} \\ -\frac{5}{3} B & = & -\frac{5}{6} m^{3} n & +\frac{10}{3} m^{3}+\frac{25}{6} m^{2} n-\frac{20}{3} m n^{2} & -\frac{50}{3} m^{2}+\frac{70}{3} m n+\frac{80}{3} n^{2} \\ -\frac{4}{3} C & = & -\frac{1}{3} m^{2} n^{2} & -\frac{8}{3} m^{3}+\frac{1}{3} m^{2} n+3 m n^{2} & +24 m^{2}-3 m n-\frac{20}{3} n^{2} \\ -2 D & = & 3 m^{3} & -21 m^{2}\end{array}$
$-D^{\prime}=\frac{\frac{3}{2} n^{3}}{-\frac{1}{6} m^{4}-\frac{4}{3} m^{3} n-\frac{10}{3} m^{2} n^{2}-3 m n^{3}-\frac{1}{2} n^{4}+4 m^{3}+\frac{28}{3} m^{2} n+\frac{61}{3} m n^{2}+9 n^{3}-\frac{27}{2} m^{2}-14 m n-\frac{229}{6} n^{2}}$

(1) $\quad+225 n+\alpha\left(\frac{1}{6} m^{3}+m^{2} n+m n^{2}+\frac{1}{6} n^{3} \quad-\frac{5}{2} m^{2}-\frac{7}{2} m n+2 n^{2}+\frac{37}{3} m-\frac{187}{6} n-75\right)+\alpha^{2}\left(-\frac{3}{2} m-\frac{3}{2} n+\right.$

(2) $-\frac{1}{3} m+\frac{8}{3} n+\alpha\left(\quad m^{2} \quad-m+8 n-1\right)+\alpha^{2}($

$\begin{array}{lll}\text { (3) } \quad+\frac{40}{3} m-\frac{320}{3} n+\alpha( & +\frac{5}{2} m n & -10 m-10 n+40) \\ \text { (4) }-\frac{160}{3} m+\frac{20}{3} n+\alpha( & m^{2} & -9 m\end{array}$

(5) $+36 m+\alpha\left(\quad-m^{2} \quad+7 m \quad-12\right)$

(6) $+18 n+\alpha\left(\quad-\frac{1}{2} n^{2}+\frac{7}{2} n-6\right)$

(7) $-\frac{13}{3} m+\frac{437}{3} n+\alpha\left(\frac{1}{6} m^{3}+m^{2} n+m n^{2}+\frac{1}{6} n^{3}-\frac{3}{2} \quad m^{2}-m n+\frac{3}{2} n^{2}-\frac{2}{3} m-\frac{89}{3} n-34\right)+\alpha^{2}\left(-\frac{3}{2} m-\frac{3}{2} n+\right.$

(twelfth equati

129. We have consequently, by means of the results just obtained,

Supp. $(\overline{5})=\kappa(\overline{5})$

$$
+N
$$

(first equation).

Supp. $(\overline{4}, 1)=\kappa(\overline{4}, 1)$

$$
+2 J+R
$$

(second equation).

Supp. $(\overline{3}, 2)=\kappa(\overline{3}, 2)$

$$
+6 K+L+3 N+20
$$

(third equation).

Supp. $(\overline{3}, 1 ; 1)=\kappa(\overline{3}, 1,1)$

$$
+D+E+F+2 G+3 J+J
$$

Supp. $(\overline{2}, 3)=\kappa(\overline{2}, 3)$

$$
+Q
$$

Supp. $(\overline{2}, 2,1)=\kappa(\overline{2}, 2,1)$

$$
+3 G+I+4 J+2 J^{\prime}
$$

(sixth equation).

Supp. $(\overline{2}, 1,1,1)=\kappa(\overline{2}, 1,1,1)$

$$
+B+4 C+4 D+D^{\prime}
$$

(seventh equation). 
Supp. $(\overline{1}, 4) \quad=\kappa(\overline{1}, 4)+\left(m-\frac{5}{3}\right)(4, N+20)$

$$
-\frac{1}{3} N+\frac{4}{3} O
$$

(eighth equation).

Supp. $(\overline{1}, 1,3)=\kappa(\overline{1}, 1,3)+\kappa(\overline{2}, 3)+\kappa .2(\overline{4}, 1)$

$$
\begin{gathered}
+(m-2)(2 P+2 Q+5 J+4 R) \\
-2 P-Q-2 R+J^{\prime}
\end{gathered}
$$

(ninth equation).

Supp. $(\overline{1}, 2,2)=\kappa(\overline{1}, 2,2)$

$$
\begin{aligned}
+(m-2)(9 K & +3 L+M+2 N+O) \\
& +3 L+2 M-2 N-O
\end{aligned}
$$

(tenth equation).

Supp. $(\overline{1}, 1,1,2)=\kappa(\overline{1}, 1,1,2)+\kappa(\overline{2}, 2,1)$

$$
\begin{aligned}
+\left(m-\frac{7}{3}\right) & (3 E+3 F+6 G+2 D+H+2 I+5 J) \\
& -2 E-2 F-G-\frac{1}{3} D+\frac{1}{3} H+\frac{5}{3} I-\frac{10}{3} J+2 D^{\prime}-J^{\prime}
\end{aligned}
$$

(eleventh equation).

Observe that

$$
G-2 E^{\prime}=0, \quad G^{\prime}-2 E=0, \quad 3 G+I+8 J=3 G^{\prime}+I^{\prime}+8 J^{\prime},
$$

relations which may be used to modify the form of the last preceding result.

Supp. $(\overline{1}, 1,1,1,1)=\kappa(\overline{1}, 1,1,1,1)+\kappa(\overline{9}, 1,1,1)$

$$
\begin{array}{r}
+\left(m-\frac{8}{3}\right)(A+2 B+4 C+3 D) \\
-\frac{4}{3} A-\frac{5}{3} B-\frac{4}{3} C-D^{\prime}
\end{array}
$$

(twelfth equation).

130. We may in these equations introduce on the right-hand sides in place of a symbol such as $\bar{p}$ the symbol $\overline{p \kappa 1}$ : for example, in the fifth equation, writing

and therefore also

$$
(\overline{2}, 3)=(\overline{2 \kappa 1}, 3)+[(\overline{2}, 3)-(\overline{2 \kappa 1}, 3)],
$$

$$
\kappa(\overline{2}, 3)=\kappa(\overline{2 \kappa 1}, 3)+\kappa[(\overline{2}, 3)-(\overline{2 \kappa 1}, 3)],
$$

the second term $\kappa[(\overline{2}, 3)-(\overline{2 \kappa 1}, 3)]$ can be expressed in terms of Zeuthen's Capitals. The remark applies to all the twelve equations; only as regards the first four of them, inasmuch as $(\overline{5 \kappa 1})=0, \ldots(\overline{3 \kappa 1}, 1,1)=0$, it is the whole original terms $\kappa(\overline{5}) \ldots \kappa(\overline{3}, 1,1)$ which are thus expressible by means of Zeuthen's Capitals. By the assistance of the formulæ (First Memoir, Nos. 69 and 73) we readily obtain

Referring to

$$
\begin{array}{ll}
\kappa(\overline{5}) & =\kappa=0 \\
\kappa(\overline{4}, 1) & =\kappa(m+n-6) \\
& =R+J^{\prime}
\end{array}
$$

(second equation). 


$$
\begin{aligned}
& \kappa(\overline{3}, 2) \quad=\kappa(-9+\alpha)=\kappa(3(n-3)+\kappa-1+1) \\
& =3 L+2 M+O \\
& \text { Referring to } \\
& \text { (third equation). } \\
& \kappa(\overline{3}, 1,1)=\kappa\left(\frac{1}{2} m^{2}+2 m n+\frac{1}{2} n^{2}-\frac{13}{2} m-\frac{13}{2} n+27-\frac{3}{2} \alpha\right) \\
& =H+2 I+D^{\prime}+J^{\prime} \\
& \text { (fourth equation). } \\
& \text { viz. } \kappa^{-1} H=\frac{1}{2} m^{2} \quad-\frac{1}{2} m+4 n \quad-\frac{3}{2} \alpha \\
& \kappa^{-1} \cdot 2 I=2 m n-6 m-8 n+24 \\
& \begin{array}{lll}
\kappa^{-1} \cdot D^{\prime}= & \cdot \frac{1}{2} n^{2} & -\frac{7}{2} n+6
\end{array} \\
& \kappa^{-1} \quad J^{\prime}=n-3 \\
& \frac{1}{2} m^{2}+2 m n+\frac{1}{2} n^{2}-\frac{13}{2} m-\frac{13}{2} n+27-\frac{3}{2} \alpha \\
& \kappa(\overline{2}, 3)=\kappa(\overline{2 \kappa 1}, 3) \\
& \text { (fifth equation). } \\
& \kappa(\overline{2}, 2,1)=\kappa(\overline{2 \kappa 1}, 2,1)+\kappa(n-3) \\
& =\kappa(\overline{2 \kappa 1}, 2,1)+J^{\prime} \\
& \text { (sixth equation). }
\end{aligned}
$$$$
\kappa(\overline{2}, 1,1,1)=\kappa(2 \kappa 1,1,1,1)+\kappa \cdot \frac{1}{2}(n-3)(n-4)
$$$$
=\kappa(2 \kappa 1,1,1,1)+D^{\prime}
$$$$
\text { (seventh equation). }
$$$$
\kappa(\overline{1}, 4)=\kappa(\overline{1 \kappa 1}, 4)+\kappa
$$$$
=\kappa(\overline{1 \kappa} 1,4)+O
$$

$$
\kappa(\overline{1}, 1,3)+\kappa(\overline{2}, 3)+\kappa 2(\overline{4}, 1)
$$

$$
\begin{array}{r}
=\kappa(1 \kappa 1,1,3)+\kappa(\overline{2 \kappa 1}, 3) \quad+\kappa(n-3) \\
+\kappa(2 \kappa 1,3) \\
+\quad \kappa(2 m+2 n-6)
\end{array}
$$$$
=\kappa(\overline{1 \kappa 1}, 1,3)+2 \kappa(\overline{2 \kappa} 1,3)+2 R+3 J^{\prime}
$$

$$
\begin{aligned}
\kappa(\overline{1}, 2,2) & =\kappa(\overline{1 \kappa 1}, 2,2)+\kappa\{3(n-3)+\kappa-1\} \\
& =\kappa(\overline{1 \kappa 1}, 2,2)+3 L+2 M
\end{aligned}
$$

$$
\begin{aligned}
\kappa(\overline{1}, 1,1,2)+\kappa(\overline{2}, 2,1) & \\
=\kappa(\overline{1 \kappa 1}, 1,1,2) & +\kappa(\overline{2 \kappa 1}, 2,1)+\kappa\left\{\frac{1}{2}(n-3)(n-4)+\delta+2 n-3 \overline{m-4}\right\} \\
& +\kappa(2 \kappa 1,2,1)+\kappa(n-3) \\
=\kappa(1 \kappa 1,1,1,2) & +2 \kappa(\overline{2 \kappa 1}, 1,2) \\
& +D^{\prime}+H+2 I+J^{\prime}
\end{aligned}
$$




$$
\begin{aligned}
\kappa(\overline{1}, 1,1,1,1)+\kappa(\overline{2}, 1,1,1) & \text { Referring to } \\
= & \kappa(\overline{1} \kappa 1,1,1,1,1)+\kappa(\overline{2 \kappa 1}, 1,1,1) \\
& +\kappa(\overline{2 \kappa 1}, 1,1,1)+\kappa \cdot \frac{1}{2}(n-3)(n-4) \\
& =\kappa(\overline{1} \kappa 1,1,1,1,1)+2 \kappa(\overline{2 \kappa} 1,1,1,1)+D^{\prime} \quad \text { (twelfth equation). }
\end{aligned}
$$

131. Hence, substituting in the expressions of the several Supplements, we have

Supp. ( $\overline{5})$

$$
\begin{array}{r}
=\quad \\
+N
\end{array}
$$

Supp. $(\overline{4}, 1)$

$$
\begin{gathered}
=\quad R+J^{\prime} \\
+2 J+R
\end{gathered}
$$

(second equation).

Supp. $(\overline{3}, 2)$

$$
\begin{aligned}
= & 3 L+2 M+O \\
+ & 6 K+L+3 N+2 O
\end{aligned}
$$

Supp. $(\overline{3}, 1,1)=H+2 I+D^{\prime}+J^{\prime}$

$$
+E+F+2 G+D+3 J+J^{\prime}
$$

(fourth equation).

Supp. $(\overline{2}, 3)$

$$
\begin{gathered}
=\quad \kappa(2 \kappa 1,3) \\
+Q
\end{gathered}
$$

Supp. $(\overline{2}, 2,1)=\kappa(\overline{2 \kappa 1}, 1,1)+J^{\prime}$

$$
+3 G+I+4 J+2 J^{\prime}
$$

Supp. $(\overline{2}, 1,1,1)=\kappa(\overline{2 \kappa} 1,1,1,1)+D^{\prime}$

$$
+B+4 C+4 D+D^{\prime}
$$

(seventh equation).

Supp. $(\overline{1}, 4)$

$$
\begin{array}{r}
=\quad(\overline{1 \kappa 1}, 4)+O \\
+\left(m-\frac{5}{2}\right)(4 N+O) \\
-\frac{1}{3} N+\frac{4}{3} O
\end{array}
$$

(eighth equation).

Supp. $(\overline{1}, 1,3)$

$$
\begin{gathered}
=\quad \kappa(\overline{1 \kappa 1}, 1,3)+2 \kappa(\overline{2 \kappa 1}, 3)+2 R+3 J^{\prime} \\
+(m-2)(2 P+2 Q+5 J+4 R) \\
-2 P-Q-2 R+J^{\prime}
\end{gathered}
$$

(ninth equation).

Supp. $(\overline{1}, 2,2)=\kappa(\overline{1 \kappa 1}, 2,2)+3 L+2 M$

$$
+(m-2)(9 K+3 L+M+2 N+0)
$$$$
+3 L+2 M-2 N-O
$$

(sixth equation).

(fifth equation).$$
\text { (sixth equation). }
$$ 
Supp. $(\overline{1}, 1,1,2)=\kappa(\overline{1 \kappa} 1,1,1,2)+2 \kappa(\overline{2 \kappa 1}, 1,2)$

$$
+H+2 I+D^{\prime}+J^{\prime}
$$$$
+\left(m-\frac{7}{3}\right)(3 E+3 F+6 G+2 D+H+2 I+5 J)
$$$$
-2 E-2 F-G-\frac{1}{3} D+\frac{1}{3} H+\frac{5}{3} I-\frac{10}{3} J+2 D^{\prime}-J^{\prime}
$$

(eleventh equation).

Supp. $(\overline{1}, 1,1,1,1)=\kappa(\overline{1 \kappa 1}, 1,1,1,1)+2 \kappa(\overline{2 \kappa 1}, 1,1,1)+D^{\prime}$

$$
\begin{array}{r}
+\left(m-\frac{8}{3}\right)(A+2 B+4 C+3 D) \\
-\frac{4}{3} A-\frac{5}{3} B-\frac{4}{3} C-2 D-D^{\prime} .
\end{array}
$$

(twelfth equation).

132. Hence finally, merely collecting the terms, we have the following expressions of the Supplements in the twelve equations respectively.

Supp. $(\overline{5})$

Supp. $(\overline{4}, 1)$

Supp. $(\overline{3}, 2)$

Supp. $(\overline{3}, 1,1)$

Supp. $(\overline{2}, 3)$

Supp. $(\overline{2}, 2,1)$

$$
=N+O
$$$$
=2 J+2 R+J^{\prime}
$$$$
=6 K+4 L+2 M+3 N+30
$$$$
=D+E+F+2 G+H+2 I+3 J+D^{\prime}+2 J^{\prime}
$$$$
=\kappa(\overline{2 \kappa 1}, 3)+Q
$$$$
=\kappa(\overline{2 \kappa 1}, 2,1)+3 G+I+4 J+3 J^{\prime}
$$

Supp. $(\overline{2}, 1,1,1)$

$$
=\kappa(\overline{2 \kappa} 1,1,1,1)+B+4 C+4 D+2 D^{\prime}
$$

Supp. $(\overline{\mathbf{1}}, 4)$

$$
=\kappa(\overline{1 \kappa 1}, 4)
$$$$
+(4 m-7) N+(2 m-1) O
$$

(first equation).

(second equation).

(third equation).

(fourth equation).

(fifth equation).

(sixth equation).

(seventh equation).

(eighth equation).

Supp. $(\overline{1}, 1,3)=\kappa(\overline{1 \kappa 1}, 1,3)+2 \kappa(\overline{2 \kappa 1}, 3)$

$$
+(2 m-6) P+(2 m-5) Q+(5 m-10) J+(4 m-8) R+4 J^{\prime} \quad \text { (ninth equation). }
$$

Supp. $(\overline{1}, 2,2)=\kappa(\overline{1 \kappa 1}, 2,2)$

$$
+(9 m-18) K+3 m L+(m+2) M+(2 m-6) N+(m-3) O \text { (tenth equation). }
$$

Supp. $(\overline{1}, 1,1,2)=\kappa(\overline{1 \kappa 1}, 1,1,2)+2 \kappa(\overline{2 \kappa 1}, 1,2)$

$$
\begin{aligned}
& +(2 m-5) D+(3 m-9) E+(3 m-9) F+(6 m-15) G \\
& +(m-1) H+(2 m-1) I+(5 m-15) J+3 D^{\prime}
\end{aligned}
$$

(eleventh equation)

Supp. $(\overline{1}, 1,1,1,1)=\kappa(\overline{1 \kappa 1}, 1,1,1,1)+2 \kappa(\overline{2 \kappa} 1,1,1,1)$

$$
+(m-4) A+(2 m-7) B+(4 m-12) C+(3 m-10) D \text {, (twelfth equation). }
$$

where I recall the remark, ante, No. 126, that in each equation the Capitals belong to the system obtained by diminishing the barred number by unity and removing the bar; (4) for the first equation, $(3,1)$ for the second, and so on.

C. VI. 
133. These are, I think, the true theoretical forms of the Supplements, viz. (attending to the signification of the Capitals) the expressions actually exhibit how the Supplement arises, whether from proper conics passing through or touching at a cusp, or from point-pairs (coincident line-pairs) or line-pairs (including of course in these terms line-pair-points). Thus, for instance, Supp. $(\overline{5})=N+O$. Referring to the explanations, First Memoir, Nos. 41 to $47, N(=\imath)$ is the number of the line-pair-points described as "inflexion tangent terminated each way at inflexion," and $O(=\kappa)$ the number of the line-pair-points described as "cuspidal tangent terminated each way at cusp," or in what is here the appropriate point of view, we have as a coincident line-pair each inflexion tangent and each cuspidal tangent. Reverting to the generation of the first equation, when the point $P$ is a point in general of the given curve, the curve $\Theta$ is the conic (5), having with the curve 5 intersections at $P$, and besides meeting it in the $2 m-5$ points $P^{\prime}$. When the point $P$ is at an inflexion, the curve $\Theta$ becomes the coincident line-pair formed by the tangent taken twice, the number of intersections at $P$ is therefore $=6$, and the inflexion is therefore (specially) a united point. Similarly, when the point $P$ is at a cusp, the curve $\Theta$ becomes the coincident line-pair formed by the tangent taken twice, the number of intersections at $P$ is therefore $=6$, and the cusp is thus (specially) a united point: we have thus the total number of special united points $=\kappa+\iota$, agreeing with the foregoing $a$ posteriori result, Supp. $(\overline{5})=N+O$.

134. Or to take another example; for the fifth equation we have

$$
\text { Supp. }(\overline{2}, 3)=\kappa(\overline{2 \kappa 1}, 3)+Q ;
$$

$Q(=2 \tau)$ is the number of the line-pair-points described as "double tangent terminated each way at point of contact," or, in the point of view appropriate for the present purpose, we have each double tangent as a coincident line-pair in respect to the one of its points of contact, and also as a coincident line-pair in respect to the other of its points of contact. Reverting to the generation of the equation, when the point $P$ is a point in general on the given curve, the curve $\Theta$ is the system of conics $(\overline{2}, 3)$ touching the curve at $P$, and having besides with it a contact of the third order; since for each conic the number of intersections at $P$ is $=2$, the total number of intersections at $P$ is $=2(\overline{2}, 3)$, and the remaining $(2 m-2)(\overline{2}, 3)$ intersections are the points $P^{\prime}$. Suppose that the point $P$ is taken at the point of contact of a double tangent; of the $(\overline{2}, 3)$ conics, 1 ( $I$ assume this is so) becomes the coincident line-pain formed by the double tangent taken twice, and gives therefore 4 intersections at $P$, the remaining $(\overline{2}, 3)-1$ conics are proper conics, giving therefore $2(\overline{2}, 3)-2$ intersections at $P$, or the total number of intersections at $P$ is $2(\overline{2}, 3)+2$ intersections ; or there is a gain of 2 intersections. As remarked (No. 96), this does not of necessity imply that the point in question is to be considered as being (specially) 2 united points; I do not know how to decide à priori whether it is to be regarded as being $\mathbf{2}$ united points or as 1 united point, but it is in fact to be regarded as being (specially) only 1 united point; and as the points in question are the $2 \tau$ points of contact of the double tangents, we have thus the number $2 \tau$ of special united points. Again, when the point $P$ is at a cusp, all the $(\overline{2}, 3)$ conics remain proper conics $((\overline{2} \kappa 1,3)=(\overline{2}, 3)$, 
First Memoir, No. 73), but each of these (quà conic touching the cuspidal tangent) has with the given curve at the cusp not 2 but 3 intersections, so that the total number of intersections at $P$ is $3(\overline{2 \kappa 1}, 3),=3(\overline{2}, 3)$, and there is a gain of $(\overline{2}, 3)=(\overline{2 \kappa 1}, 3)$ intersections. Each cusp counts (specially) as $(\overline{2 \kappa 1}, 3)$ united points, and together the cusps count as $\kappa(\overline{2 \kappa 1}, 3)$ united points; we have thus the total number $\kappa(\overline{2 \kappa 1}, 3)+2 \tau$ of special united points, agreeing with the expression, Supp. $(\overline{2}, 3)=\kappa(\overline{2 \kappa 1}, 3)+Q$.

135. As appears from the preceding example, or generally from the remark, ante, No. 96, I have not at present any à priori method of determining the proper numerical multipliers of the Capitals contained in the expressions of the several Supplements. I will only further remark, that the reason is obvious why (while in the first seven equations the multipliers are mere numbers) in the eighth and following equations the multipliers are linear functions of $m$; in fact in these last equations the barred symbol is $\overline{1}$, that is, when $P$ is a point in general on the given curve, each of the conics which make up the curve $\Theta$ has with the given curve noi a contact of any order, but an ordinary intersection at $P$. Imagine a position of $P$ for which one of these conics becomes a coincident line-pair; this regarded as a single line has with the given curve $(m-\alpha)$ ordinary intersections $(\alpha$ a number, $=4$ at most, depending on the contacts which the line may have with the curve); for each of the $m-\alpha$ points, taken as a position of $P$, one of the conics which make up the curve $\Theta$ becomes the coincident line-pair, and there are in respect of this conic two intersections at $P$ instead of one intersection only. We have thus in respect of the particular coincident line-pair a group of $(m-\alpha)$ special united points, viz. these are the $m-\alpha$ ordinary intersections of the coincident line-pair regarded as a single line with the given curve, and we thus understand in a general way how it is that the order $m$ of the given curve enters into the expressions of the multipliers of the several Capitals in the last five equations. The object of the present Memoir was, however, the $d$ posteriori derivation of the expressions (ante, No. 132) of the twelve Supplements; and having accomplished this, but being unable to discuss the results with any degree of completeness, I abstain from a further discussion of them. 